\title{
Carcinoma midollare della tiroidea trattato con vandetanib: fattori predittivi di risposta duratura
}

\author{
Maria Grazia Castagna ${ }^{1}$
}

Accettato: 6 agosto 2020 / Pubblicato online: 29 ottobre 2020

(c) The Author(s) 2020

Commento a:

Medullary thyroid cancer treated with vandetanib: predictors of a longer and durable response. L. Valerio, V. Bottici, A. Matrone, P. Piaggi, D. Viola, V. Cappagli, L. Agate, E. Molinaro, R. Ciampi, A. Tacito, T. Ramone, C. Romei, R. Elisei. Endocr Relat Cancer (2020) 27(2):97-110

Il carcinoma midollare della tiroide (CMT) si sviluppa a partire dalle cellule $\mathrm{C}$ della tiroide e rappresenta il 5-10\% dei tumori della tiroide. La sopravvivenza globale a 10 anni è di circa il $75 \%$ ma si riduce significativamente al $40 \%$ nei soggetti con malattia metastatica locoregionale o a distanza [1]. Per questi pazienti sono oggi disponibili terapie a bersaglio molecolare come il vandetanib e il cabozantinib, i due farmaci ad oggi approvati dalla FDA e dall'EMA per il trattamento del paziente con CMT localmente avanzato o metastatico [2].

Lo studio di Valerio e collaboratori [3] ha avuto come obiettivo quello di individuare fattori in grado di predire la durata della terapia con vandetanib in pazienti con CMT. Uno dei limiti di queste terapie è, infatti, la durata di risposta al farmaco. In un numero significativo di pazienti è possibile osservare, dopo un periodo variabile di risposta, lo sviluppo di una resistenza al farmaco con conseguente ripresa della progressione di malattia.

Nello studio di Valerio e colleghi [3] sono stati valutati retrospettivamente 79 pazienti con CMT trattati con vandetanib. Venticinque pazienti sono stati trattati per un periodo di tempo inferiore a 12 mesi, 54 sono per un periodo di tempo superiore o uguale a 12 mesi e, infine, 24 di questi ultimi

M.G. Castagna

mariagrazia.castagna@unisi.it

1 Dipartimento di Scienze Mediche, Chirurgiche e Neuroscienze, Università di Siena, Siena, Italia sono stati trattati per un periodo di tempo uguale o superiore a 48 mesi (durata del trattamento: breve, lunga e molto lunga). La sopravvivenza libera da progressione di malattia dei pazienti con durata del trattamento $\geq 12$ mesi e $\geq 48$ mesi è stata significativamente maggiore rispetto a quella riportata nello studio registrativo del vandetanib [4].

Dal confronto tra i due gruppi principali $(<12$ mesi e $\geq 12$ mesi) emergeva come nel gruppo con una maggiore durata al trattamento, $\mathrm{i}$ pazienti erano più frequentemente naïve per i TKI (90,7 versus $72 \%$ ) mentre quelli con risposta più breve erano stati già precedentemente trattati (28 versus $9,2 \%, p=0,04)$. In aggiunta, i pazienti trattati per più di 12 mesi avevano una più giovane età sia alla diagnosi che all' $i-$ nizio del trattamento con vandetanib e tale differenza diventava statisticamente significativa quando veniva considerato il sottogruppo di pazienti trattati per più di 48 mesi. Fattori predittivi di una migliore risposta al trattamento risultavano essere la giovane età, l'essere stati trattati per la presenza di sintomi e non per progressione di malattia e l'aver sviluppato eventi avversi duranti il trattamento. Nel sottogruppo di pazienti trattati per più di 48 mesi, l'aver iniziato la terapia per la presenza di sintomi piuttosto che per progressione di malattia, è risultato l'unico fattore associato con la risposta al trattamento.

In conclusione, i dati di questo studio dimostrano come iniziare il trattamento con vandetanib in soggetti giovani, in buono stato di salute e quando non c'è ancora una chiara evidenza di progressione di malattia potrebbe garantire una risposta al trattamento più duratura nel tempo. I risultati di questo studio, sebbene meritevoli di conferma, per la prima volta mettono in discussione quello che sono le attuali raccomandazione per l'inizio del trattamento con TKI in soggetti con carcinoma avanzato della tiroide. Infatti, considerando che il CMT ha un lento accrescimento e un'ottima qualità di vita e che tali farmaci hanno un'azione citostatica e non 
citotossica, l'indicazione è di iniziare il trattamento quando si evidenzia una chiara progressione di malattia. I risultati di Valerio e collaboratori [3] suggeriscono la necessità di rivalutare la tempistica dell'inizio del trattamento, nel tentativo di implementare l'efficacia terapeutica dei TKI e migliorare la sopravvivenza libera da malattia nei pazienti con CMT.

Funding Note Open access funding provided by Università degli Studi di Siena within the CRUI-CARE Agreement.

Nota della casa editrice Springer Nature rimane neutrale in riguardo alle rivendicazioni giurisdizionali nelle mappe pubblicate e nelle affiliazioni istituzionali.

Open Access This article is licensed under a Creative Commons Attribution 4.0 International License, which permits use, sharing, adaptation, distribution and reproduction in any medium or format, as long as you give appropriate credit to the original author(s) and the source, provide a link to the Creative Commons licence, and indicate if changes were made. The images or other third party material in this article are included in the article's Creative Commons licence, unless indicated otherwise in a credit line to the material. If material is not included in the article's Creative Commons licence and your intended use is not permitted by statutory regulation or exceeds the permitted use, you will need to obtain permission directly from the copyright holder. To view a copy of this licence, visit http://creativecommons.org/licenses/by/4.0/.

\section{Bibliografia}

1. Lakhani VT, You YN, Wells SA (2007) The multiple endocrine neoplasia syndromes. Annu Rev Med 58:253-265

2. Wells SA Jr, Robinson BG, Gagel RF et al (2012) Vandetanib in patients with locally advanced or metastatic medullary thyroid cancer: a randomized, double-blind phase III trial. J Clin Oncol 30(2):134-141

3. Valerio L, Bottici V, Matrone A et al (2020) Medullary thyroid cancer treated with vandetanib: predictors of a longer and durable response. Endocr-Relat Cancer 27(2):97-110

4. Wells SA Jr, Robinson BG, Gagel RF, Dralle H, Fagin JA, Santoro M, Baudin E, Elisei R, Jarzab B, Vasselli JR, Read J, Langmuir P, Ryan AJ, Schlumberger MJ (2012) Vandetanib in patients with locally advanced or metastatic medullary thyroid cancer: a randomized, double-blind phase III trial. J Clin Oncol 30(2):134-141. https://doi.org/10.1200/JCO.2011.35.5040 